\title{
p53 expression in anaplastic carcinomas arising from thyroid papillary carcinomas
}

\author{
X Matias-Guiu, M Cuatrecasas, E Musulen, J Prat
}

Department of

Pathology, Hospital de

la Santa Creu i Sant

Pau, Autonomous

University of

Barcelona, Spain

X Matias-Guiu

M Cuatrecasas

E Musulen

J Prat

Correspondence to: Xavier Matias-Guiu

Department of Pathology,

Hospital de la Santa Creu

Sant Pau, Av. San Antonio

Ma Claret 167, 08025

Barcelona, Spain.

Accepted for publication 27 October 1993

\begin{abstract}
Aim-To study the expression of p53 tumour suppressor gene in anaplastic carcinomas arising from thyroid papillary carcinomas.

Methods-Formalin fixed, paraffin wax embedded tissues from four cases of anaplastic carcinomas associated with thyroid papillary carcinomas were studied by immunohistochemistry with two different p53 monoclonal antibodies.

Results-The anaplastic component showed nuclear immunostaining in two cases, but not in the other two. In all cases the papillary carcinoma component was negative.

Conclusion-The results support the hypothesis that p53 stimulates tumour progression in thyroid tumours.
\end{abstract}

( Clin Pathol 1994;47:337-339)

The tumour suppressor gene $\mathrm{p} 53$ is supposed to be involved in the process of neoplastic transformation in many human tumours. ${ }^{1-4}$ In the thyroid gland p53 mutations have been reported to occur in anaplastic carcinoma more often than in well differentiated carcinoma. $\mathrm{p} 53$ mutations in thyroid tumours may be associated with aggressive behaviour and loss of differentiation..$^{5-8}$ To provide additional information about this putative tumour pro-

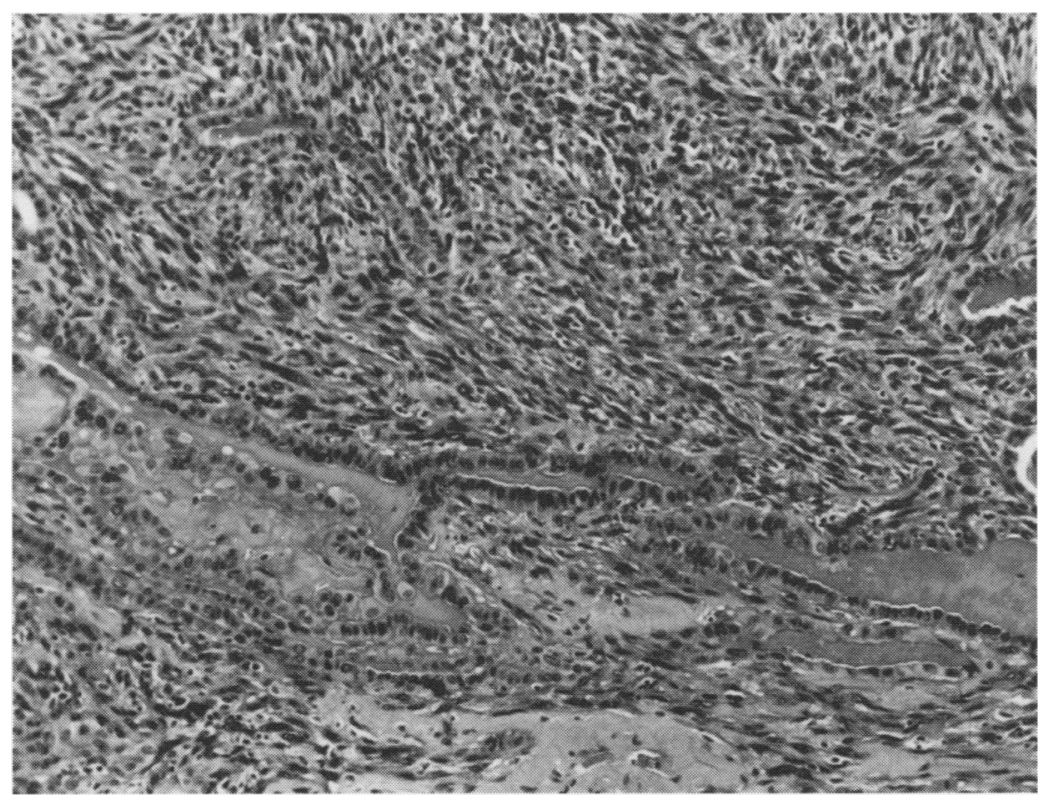

Figure 1 Focus of anaplastic carcinoma is admixed with remnants of a pre-existing thyroid papillary carcinoma (haematoxylin and eosin). gression role for $\mathrm{p} 53$ in the thyroid gland we have examined immunohistochemically four cases of anaplastic carcinoma arising from papillary carcinoma. The specific aim of the study was to evaluate the expression of p53 and compare the staining pattern in both components.

\section{Methods}

Four cases of anaplastic carcinoma in association with thyroid papillary carcinoma were studied. The cases were obtained from the files of this hospital in a review of 21 cases of anaplastic carcinoma of the thyroid. Clinical charts, histological slides, and paraffin wax embedded blocks of tissue were available in all cases. In all the specimens both components were closely admixed, and in some fields foci of anaplastic carcinoma merged imperceptibly with others of papillary carcinoma (fig 1).

For immunohistochemistry, two monoclonal antibodies were used with the avidinbiotin-peroxidase method: Ab2 Pab 1801 (Oncogene Science, Uniondale, New York; dilution 1 in 50) and BP53-12-1 (Biogenex, San Ramon, California; prediluted). To increase immunostaining the sections were treated with an antigen retrieval solution (10 $\mathrm{mM}$ citric acid monohydrate, $\mathrm{pH} 6 \cdot 0$, adjusted with $2 \mathrm{~N} \mathrm{NaOH}$ ), heated in a microwave oven at high power for five minutes, three times. The incubation with the antibodies was performed overnight at room temperature. Following the peroxidase diaminobenzidine (DAB) colorisation, the staining was enhanced by immersing the slides in $0.5 \%$ $\mathrm{CuSO} 4 / 0.9 \% \mathrm{CINa}$ solution for three minutes. Sections of colon adenocarcinomas known to show p53 mutations were used as positive controls. Normal connective tissue was taken as an internal negative control in all cases.

\section{Results}

There was a good correlation between the immunohistochemical results of both antibodies. Strong nuclear staining was found throughout the anaplastic component of two cases; the anaplastic areas of the remaining two were negative. The number of positive tumour cells was high in one tumour $(80 \%)$ but low in the second (20\%). In all four cases the papillary carcinoma component was negative (figs 2 and 3 ). In two cases a faint cytoplasmic staining without nuclear staining was seen in occasional cells with the $\mathrm{Ab} 2 \mathrm{pAb}$ 1801 antibody but not with the Bp53-12-1 


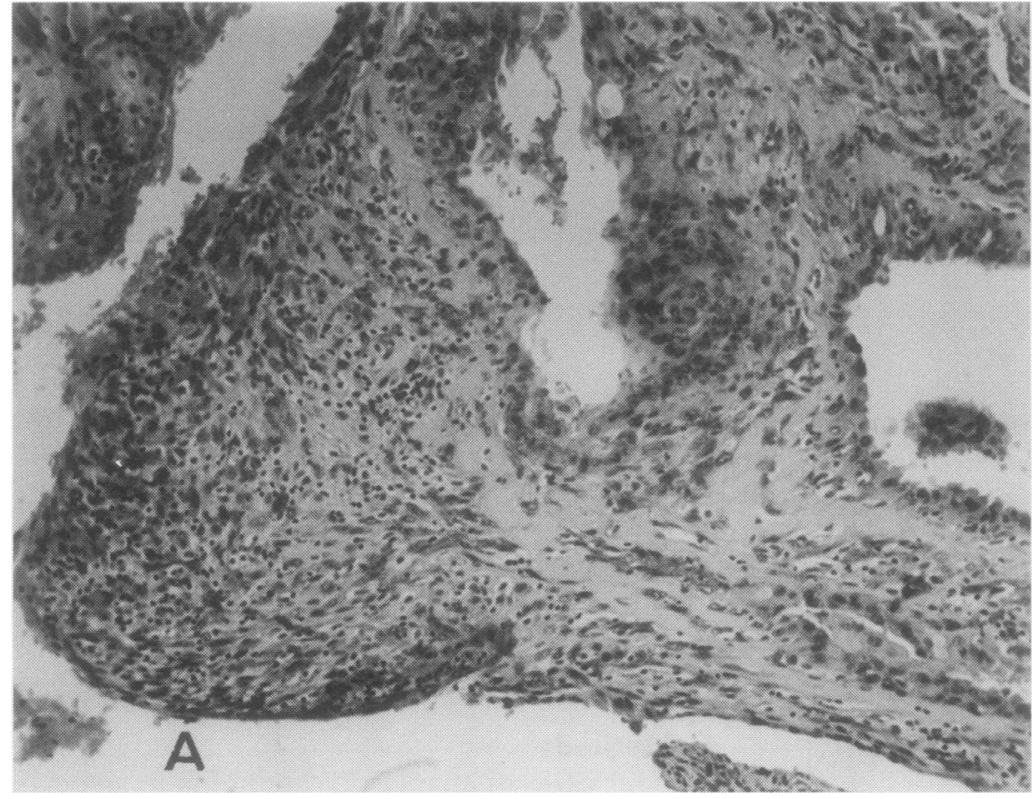

Figure 2 Papillary thyroid carcinoma associated with anaplastic areas (haematoxylin and eosin).

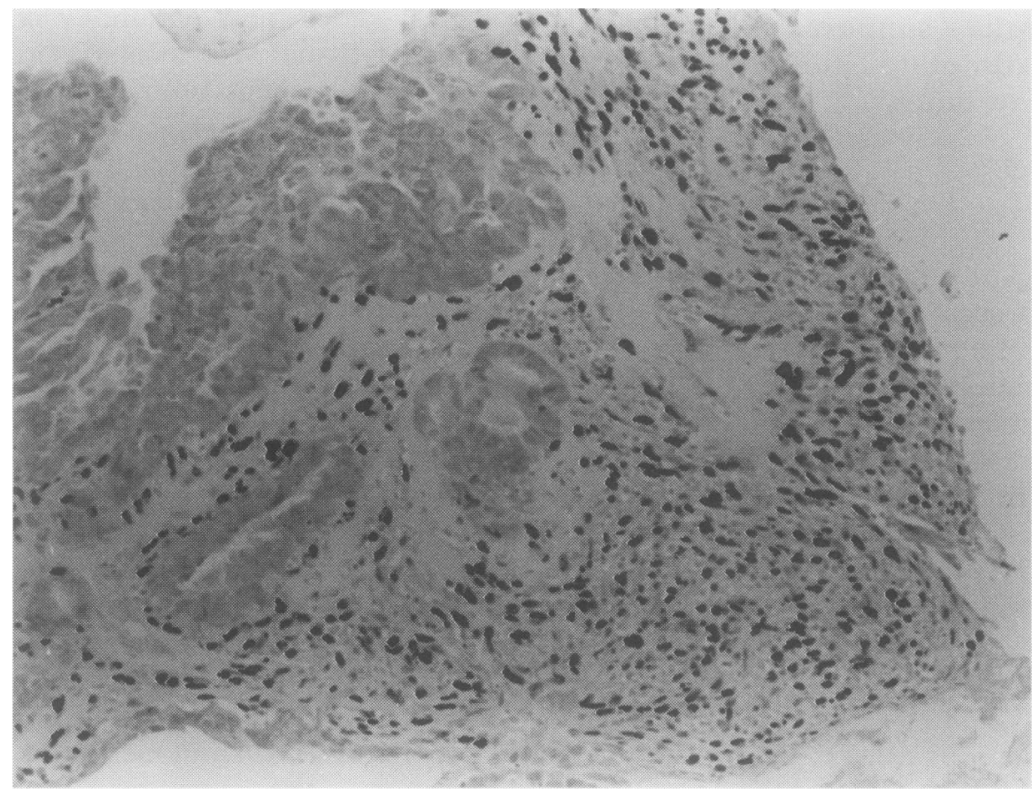

Figure 3 Anaplastic carcinoma arising from thyroid papillary carcinoma. Notice nuclear immunostaining for $p 53$ in the anaplastic component, whereas the papillary areas are negative (avidin-biotin-peroxidase).

antibody. This pattern was not regarded as positive.

\section{Discussion}

p53 is a nuclear phosphoprotein that has a role in the regulation of normal cell proliferation. ${ }^{1}$ It is encoded by a tumour suppressor gene located on the short arm of chromosome 17. Inactivation of p53 tumour suppressor gene is one of the most common genetic abnormalities found in human neoplasia. ${ }^{2}$ Missense mutations are the most common expression of $\mathrm{p} 53$ gene inactivation and lead to the production of an abnormal protein with longer half life than the normal p53 phosphoprotein. The mutated p53 protein can then be detected by immunohistochemistry. ${ }^{3}$
Although molecular biology techniques are the most accurate way of identifying p53 inactivation, immunohistochemistry can also be a very useful and indirect method of assessing p53 activity and has some value in studying different components of the tumours in their appropriate morphological context. Although a $96 \%$ concordance between positivity by immunohistochemistry and the presence of missense mutations has been reported in some series, false negative and false positive results can occur as a result of technical problems or abnormal p53 protein stabilisation or degradation..$^{9}{ }^{10}$ Furthermore, nonsense mutations, deletions, and splice site mutations cannot be immunostained. ${ }^{4}$

There is some evidence to suggest that p53 gene inactivation might have a role in the genesis of thyroid tumours, particularly in the progression to anaplastic carcinoma. By using molecular biology techniques, such as single strand conformational polymorphism (SSCP) and direct sequencing analysis, several authors have demonstrated a high prevalence of p53 mutations in anaplastic (22-86\%) and in poorly differentiated carcinomas $(25 \%)$, in contrast to normal thyroid $(0 \%)$, follicular adenomas $(0 \%)$, papillary carcinomas $(0 \%)$ and follicular carcinomas $(0-9 \%) .^{5-8}$ Conversely, three studies have detected overexpression of p53 by immunohistochemistry in $18-83.3 \%$ of cases of anaplastic carcinomas, but also in $0-11 \%$ of papillary carcinomas, $14 \cdot 3 \%-20 \%$ of well differentiated follicular carcinomas, and $16 \cdot 1-40 \cdot 9 \%$ of poorly differentiated carcinomas. ${ }^{11-13}$ Although there are some discrepancies between the results of molecular biology and immunohistochemistry, it seems obvious that p53 is more prevalent among anaplastic rather than well differentiated papillary and follicular carcinomas.

To provide further data in this respect, we used immunohistochemistry to study the expression of p53 in four anaplastic carcinomas associated with papillary thyroid carcinomas. In these neoplasms both types of tumour merged imperceptibly and anaplastic carcinoma seemed to have originated from pre-existing well differentiated papillary carcinoma. ${ }^{14}$ We found positive nuclear immunostaining in the anaplastic component of two cases; the papillary carcinoma areas were negative in all cases. These results are consistent with other previously reported data ${ }^{78}$ and suggest a role for p53 in tumour progression. Nakamura et al have recently demonstrated (by RNA protection analysis and DNA sequencing) the presence of p53 mutations in two out of nine anaplastic carcinomas and their absence in coexisting foci of papillary carcinomas. ${ }^{7}$ Moreover, Donghi et al used sequence analysis and immunohistochemistry to show the presence of mutations in two anaplastic and one poorly differentiated carcinomas. ${ }^{8}$ The associated well differentiated areas were negative. Our study lends support to the hypothesis that $\mathrm{p} 53$ probably has a role in the progression of thyroid tumour to anaplastic carcinoma. 
This work was supported by a grant CIRIT AR91-232.

1 Finlay CA, Hinds PW, Levine AJ. The $\mathrm{p} 53$ protooncogene can act as a suppressor of transformation. Cell 1989; can act as a
57:1083-93.

2 Crawford LV, Pim DC, Lamb P. The cellular protein p53 in human tumours. Mol Biol Med 1984;2:261-72.

3 Bartek J, Iggo R, Gannon J, Lane DP. Genetic and immunochemical analysis of mutant $\mathrm{p} 53$ in human breast cancer cell lines. Oncogene 1990;5:893-9.

4 Kupryjanczyk J, Beauchamp R, Bell D, Yandell DW, Tho A. p53 gene alterations and p53 protein expression in ovarian cancers. Mod Pathol 1993;6:75A.

5 Ito $T$, Seyama $T$, Mizuno T, Tsuyama N, Hayashi $T$, Hayashi $Y$, et al. Unique association of p53 mutations with undifferentiated but not with differentiated carcinomas of the thyroid gland. Cancer Res 1992;52:1369-71.

6 Fagin JA, Matsuo K, Karmakar A, Chen DL, Tang S Koeffler HP. High prevalence of mutations of the p53 gene in poorly differentiated human thyroid carcinomas. f Clin Invest 1993;91:179-84.

7 Nakamura T, Yana I, Kobayashi T, Shin E, Karakawa K, Fujita $S$, et al. p53 gene mutations associated with anaplastic transformation of human thyroid carcinomas. Fpn f Cancer Res 1992;83:1293-8.
8 Donghi R, Longoni A, Pilotti S, Michieli P, Della Porta G, Pierotti MA. Gene p53 mutations are restricted to poorly differentiated and undifferentiated carcinomas of the thyroid gland. 7 Clin Invest 1993;91:1753-60.

9 Wynford-Thomas D. p53 in tumour pathology: Can we trust immunocytochemistry? F Pathol 1992;166:329-30.

10 Yandel DW, Thor AD. p53 analysis in diagnostic pathology. Biologic implications and possible clinical applications. Diagn Mol Pathol 1993;2:1-3.

11 Dobashi Y, Sakamoto A, Sugimura H, Mernyei M, Mori $M$, Oyama $T$, et al. Overexpression of $p 53$ as a possible prognostic factor in human thyroid carcinoma. $\mathrm{Am}$ Surg Pathol 1993;17:375-81.

12 Carr $K$, Heffes $C$, Jin $L$, Davenport $R$, Thompson $N$, Sisson $\mathrm{J}$, et al. Immunohistochemical analysis of papillary and anaplastic thyroid carcinomas utilizing antibodies to p53, PCNA and Ki-67. Mod Pathol 1993;6: 37A.

13 Soares $P$, Cameselle-Tejeiro J, Sobrinho-Simoes $M$ Immunohistochemical detection of p53 and PCNA in differentiated, poorly differentiated and undifferentiated carcinomas of the thyroid. Pathol Res Pract 1993; 189:813.

14 Rosai J, Saxen EA, Woolner L. Undifferentiated and poorly differentiated carcinoma. Semin Diagnost Patho 1985;2:123-36. 